\title{
A Network-based Approach for Assessing Co-Operating Manned and Unmanned Systems (MUMS)
}

\author{
Dr. Lora G. Weiss \\ Georgia Tech Research Institute \\ $25014^{\text {th }}$ St. NW \\ Atlanta, GA 30334-0822 \\ 404-407-7611, Lora.Weiss@gtri.gatech.edu
}

\begin{abstract}
Traditionally, robots have been programmed to do precisely what their human operators instruct them to do, but more recently, they have become more sophisticated, intelligent, and autonomous. Once they reach a sufficiently high level of intelligent autonomy, they can support more collaborative interactions with each other and with people. As robots become more and more intelligent, we will begin designing systems where robots interact with humans, rather than designing robots that are commanded by people with continual oversight. One approach to assessing how humans and robots will interact in the future is to frame the problem as a collection of intelligent nodes. Multiple, collaborating, and interacting manned and robotic systems can be represented as a collection of dynamic, interacting nodes. This paper develops preliminary metrics to support understanding the extent of preferential attachment that would arise in a system of cooperating manned and unmanned systems (MUMS). The metrics seek to help explain if attachments are localized to specific situations or if they are more pervasive throughout a MUMS society.
\end{abstract}

\section{General Terms}

Algorithms, Measurement, Performance, Design.

\section{Keywords}

Robotics. Clustering. Dynamic Metrics.

\section{INTRODUCTION}

MUMS is an acronym for Manned and Un-Manned Systems. Unmanned Systems may include unmanned ground vehicles (robots), unmanned air vehicles, unmanned undersea vehicles, and unmanned sea surface vehicles. We often use the terms robots and unmanned vehicles interchangeably. Co-Operating MUMS is the concept of people and machines cohabitating, cooperating, or interacting in a shared or common environment. See Figure 1. This paper develops preliminary metrics to assess MUMS interactions in a meaningful way from an overall network and system perspective.

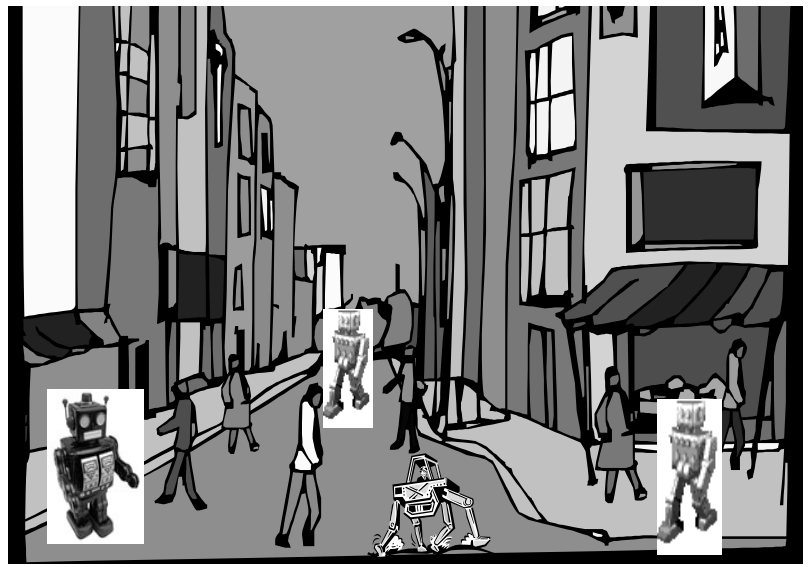

Figure 1. Graphical Concept of Many Co-Operating Manned and Unmanned Systems

These methods support analyzing dynamic MUMS interactions, where mobile robots interact and co-operate with people, but without continual oversight by an operator commanding the robots, and with multiple levels of interaction between the manned and unmanned systems. The levels of interaction include: assistance of unmanned systems to humans, independence of humans and unmanned systems (separate tasks in shared spaces), and voluntary, un-commanded assistance between humans and unmanned systems.

Our approach treats MUMS as a dynamic network of dynamic nodes, where the nodes are a mix of people, computers, and robots. Both the nodes and the edges are dynamic. The nodes are dynamic since some of them change or move in space and time, while the edges are dynamic since the connections between the nodes change as a function of time (e.g., the location of an individual, information on their activities, identification of their normal modes of behaviors, etc.). Describing these dynamic interactions poses several challenges that traditional static network architectures do not fully address.

Social robotics is an expanding research area [GT]. Kiesler and Hinds [Kie] have identified that people seem to perceive autonomous robots differently than most other computer technologies. People's mental models of robots are usually more

Permission to make digital or hard copies of all or part of this work for personal or classroom use is granted without fee provided that copies are not made or distributed for profit or commercial advantage, and that copies bear this notice and the full citation on the first page. To copy otherwise, to republish, to post on servers or to redistribute to lists, requires prior specific permission and/or a fee. PerMIS'10, September 28-30, 2010, Baltimore, MD, USA. Copyright (c) 2010 
anthropomorphic than systems being developed. They also confirm that autonomous robots are becoming more and more able to make their own decisions. Hinds, et al. [Hin] evaluate the effects of robot appearance on human-robot collaborations. They also evaluate status (e.g., subordinate, peer, and supervisor) on human-robot relationships. Breazeal [Bre] describes how to design robots to interact with people, where they learn in the human environment and learn to become social partners.

An equally impressive amount of research has been conducted in the area of understanding social and technical networks [New]. Research spans areas such as random graph theory from early on [Erd], to understanding the small world problem [Tra], and modeling the author-chain of scientific papers [deS]. Structures have emerged of the world-wide web [Bro], massive graphs [Aie], small-world networks [Wat], and scale-free networks [Bar]. Newman [New2] provides a reflective account of various networks, including social, information, technological, and biological networks in the real world. Capturing properties and creating models of the interactive nodes and the associated dynamics is an ever growing research area. The coupling of social-technical systems, as with MUMS, is yet one more application of the need for a robust capability to analyze these collections of nodes.

This research on social robotics and system networks leads to the need to start evaluating how robots will be operating in the context of a social system. We wonder how they will operate within our society, beyond one-on-one interactions with people. Will they exhibit preferential attachment or cluster by type? Are these behaviors more pervasive throughout a MUMS societal network? It is natural to expect that such attachments will result, since in social networks, certain individuals acquire more social links, e.g., if a person is older, famous, or just more personable, this person will have more connections. It will be interesting to learn if older robots will increase their connectivity to people or other robots as they age. Another question is whether or not the robots will "socialize" to become more knowledgeable and increase their situational awareness. People call this business networking, but will robots learn to do this?

Sociological networks support many dynamic interactions, yet are laden with numerous uncertainties. For example, in social networks, individuals can find relatively short paths to other individuals. This is despite having limited information about the structure of the network. Individuals also categorize themselves and each other into socially meaningful groups, often because they have the same occupation, interest, or geographical location. Without a means to estimate social distance or relationships, individuals are often unable to move information, such as a message, closer to a remote place or contact. By making social groupings, social distance feeds back into the structure of the social network, and the result is that messages, for example, make successively more precise jumps as they near their destination.

From a sociological perspective, network interactions fall into three main categories. See Table 1. Although these are worded from a sociological perspective, they apply to Co-operating MUMS networks as well. A key rationale for this research is the need to capture these notions with a rigorous approach, where metrics can be computed for these interacting systems and where performance can be quantitatively assessed across the dynamic network of nodes.

Table 1. Interactions within Complex Systems, from a Sociological Perspective

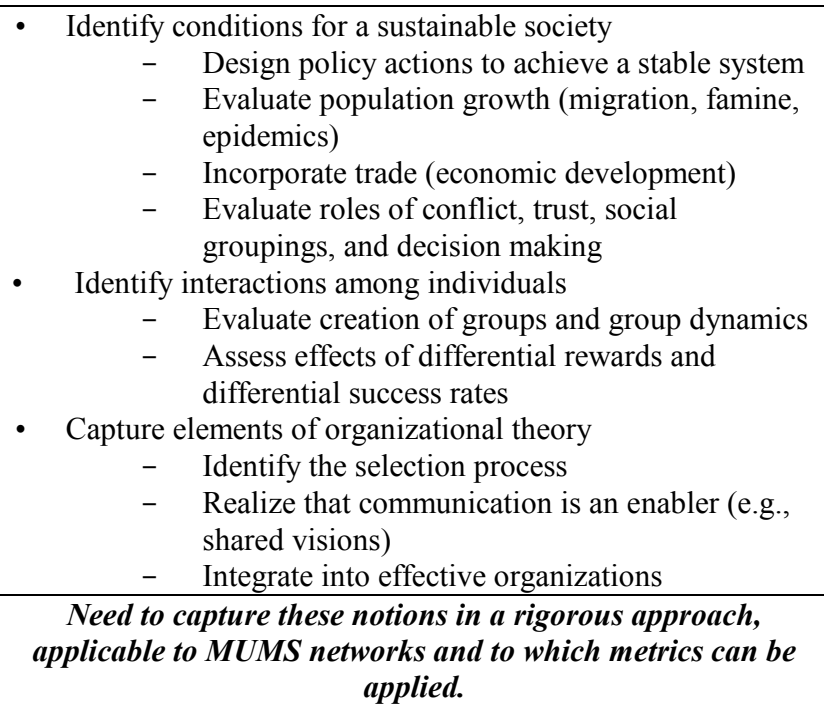

In contrast to social networks, mathematical graphs are networks of well defined nodes whose connections are often similar and known. Capturing the dynamics of Co-operating MUMS networks poses several challenges that traditional network architectures currently do not. Traditional approaches to understanding networks of nodes often involve graph theory, lattice analysis, and centralized/decentralized analyses. Elements of current approaches also include static analyses, empirical analyses, qualitative assessments, limiting assumptions, and non-integrated approaches. For MUMS applications, the dynamic network is evolving in-situ in an unplanned and decentralized manner, so that an integrated framework that enables analysis of more complex interactions is necessary.

For interacting manned and unmanned systems that are collaborating in an un-commanded environment, these systems assemble, interact, process information, and separate from one another. For networks with large numbers of nodes, there can be thousands of such interactions. These interactions, in some ways, parallel biochemical interactions within cellular transcription networks more so than traditional mathematical or social networks. Because biological networks have evolved to perform specific functions, they are far from random [Alo]. As evolution continually adjusts these networks, they converge to a set of nodal interactions that obey general design principles.

Some of the biological design principles directly relate to MUMS networks and support the development of an initial framework for modeling, assessing, and understanding the dynamic interactions [Wei]. The features of biological transcription networks that are applicable to networks of manned and unmanned systems include the reuse of a small set of network motifs (patterns of node interactions), robustness to component tolerances, constrained interactions, and modularity. Although communications between

Permission to make digital or hard copies of all or part of this work for personal or classroom use is granted without fee provided that copies are not made or distributed for profit or commercial advantage, and that copies bear this notice and the full citation on the first page. To copy otherwise, to republish, to post on servers or to redistribute to lists, requires prior specific permission and/or a fee. PerMIS'10, September 28-30, 2010, Baltimore, MD, USA. Copyright (c) 2010 
genes may be different than for MUMS, information is conveyed between nodes and a response is generated depending on the strength or content of a stimulating signal which is the input function that triggers parts of the network into action.

A key to the understanding how to measure smaller motifs within larger collections of robotic interactions is to define and develop metrics that are reflective of interactions within a dynamic network. This paper presents a first look at expanding mathematical, sociological, and biological network analysis methods to measure and understand issues such as whether or not nodes exhibit preferential attachment to other nodes (as in society), or if preferential attachment is better than diverse attachment for robots, or if will nodes compete for links at the expense of other nodes (robots).

\section{MUMS NETWORKS - The Components}

A MUMS network consists of a collection of multiple, interacting nodes (people and robots). Each node can accomplish several tasks, but not every node can do everything. Its capabilities are determined by its sensing systems (e.g., eyes and ears for humans, lasers and radars for robots), its assets (e.g., feet and arms for humans, power and wheels for robots), its functionality, and its dynamics.

The nodes are assumed to be intelligent and autonomous and can interact in a complex external environment. They can measure internal and external states. For example, their internal state includes knowledge of their remaining fuel (or hunger for humans) or damaged components (a cracked gear for robots, a broken arm for humans). External states include physical parameters such as temperature, current position, physical contact, or communications. Communications (both overt and inferred) are extremely important for these networked systems. The information content relayed between MUMS nodes provides the stimulus for a node to react differently than in its current state. Nodes respond to these signals and act upon them. To understand a MUMS network, it is important to understand these interactions.

A Co-Operating MUMS Network consists of a collection of nodes and edges. Each node (person or robot) is different and has its own dynamics. Nodes interact with other nodes in different ways and across different time-scales. Not all nodes interact with each other. When nodes interact, they may do so only once or quite often, and their interactions may be different each time. For MUMS networks, the nodes are the people and the unmanned, robotic systems.

The edges in a MUMS network are the interactions between nodes, where one node directly influences another node. Edges can be directed or non-directed. Directed edges originate at one node and terminate at another node with the information flowing in one direction. Two nodes can be connected with a directed edge or with a bi-directional edge, e.g., human can ask a robot for directions, and the robot can respond with information to the person. For MUMS networks, we assume all edges are directed and that the direction of the edge indicates the direction of influence. However, not all nodes are connected to all other nodes, i.e., this is not a fully connected network. See Figure 2.

Permission to make digital or hard copies of all or part of this work for personal or classroom use is granted without fee provided that copies are not made or distributed for profit or commercial advantage, and that copies bear this notice and the full citation on the first page. To copy otherwise, to republish, to post on servers or to redistribute to lists, requires prior specific permission and/or a fee. PerMIS'10, September 28-30, 2010, Baltimore, MD, USA. Copyright (c) 2010

\subsection{General Metrics}

The approach to MUMS analysis investigates relationships within dynamically evolving networks based on concepts in Table 1. A key to the success of this approach is development of concepts that enable robust interactions to be quantitatively measured and behavioral concepts to be analytically assessed. This section discusses several metrics that are reflective of interactions within a dynamic network.

Bow-Tie Diagrams - "Bow-tie" diagrams [Bro, et al.] were initially developed for world-wide-web applications, but have been shown to be applicable to areas such as metabolic networks, food webs, and email networks [New]. The "bow-tie" diagram represents a central, connected component (e.g., a subway station or city center) and integrates disconnected components (e.g., robots or individuals) that link into and out of the system as a function of time. There are other external components, called tendrils, which affect the system dynamics (e.g., a street vendor) and are involved in the interplay of the network. Such a diagrammatic representation of entities is indicative of MUMS behaviors and may provide a useful for analysis.

Scale-Free Networks - To capture the relationship between the complexity of the system, notions such as emergence and uncertainty must be considered. The computer science community recently identified the internet as a scale-free network. This roughly means that as entities are added to the network, the probability that they will interact with others is the same as for existing entities. From a MUMS analysis perspective, this is important. If connectedness is a measure of behavioral interactions, then introducing an additional robot to the network may not necessarily increase complexity. Understanding these dynamics may yield valuable insights into the large-scale structure needed for effective MUMS operations and parallels similar research in areas such as metabolic networks of organisms and biochemistry.

Clustering Coefficient and Nodal Affinity - Nodal affinity aims to determine if entities within a network will migrate to or be attracted to other entities in the network (e.g., will Fed Ex robots interact with UPS robots). In social networks, certain nodes prefer to attach themselves to the more popular nodes. This is termed preferential attachment or nodal affinity. Capturing nodal relationships and connectivity can reveal unique behaviors within a dynamical network of systems, and would be highly applicable 
MUMS relationships. To capture the connectivity, a clustering coefficient can be computed [Wat]. High clustering means there is a higher chance that two nodes will interact with each other if there is another node near each of them (i.e., they have a common friend). However, too much interaction may be inefficient and may be detectable. For example, if all the Fed Ex robotic delivery trucks clustered together, they would not efficiently deliver packages. A clustering coefficient is one approach to mathematically measure interactions between nodes.

Clustering and Complexity - The clustering phenomenon limits a system from becoming computationally complex, in that similar nodes may group together. The probability that a new node will connect to an existing node may not be uniform, but there may possibly be a higher probability that it will be linked to a node with several other connections.

Social Groupings - In social networks, individuals find the shortest paths to others. This is despite having limited information about the structure of the network. If a person wants to deliver a message by having it handed from one person to the next, it turns out that each person will hand it to someone with whom they are connected. Without a means to estimate social distance, individuals are unable to move a message closer to the target. By making social groupings, social distance feeds back into the structure of the network. The result is that messages make more precise jumps as they near their target. A mathematical notion of social distance can be equally applied to MUMS networks, but where the robots are provided means to measure distances and as such progress to their destination.

Random Networks: For a network with $N$ nodes and $E$ edges, then there are $N(N-1) / 2$ possible pairs of nodes that can be connected by an edge. Each edge can point in one of two directions, for a total of $N(N-1)$ possible directed edges between nodes. If $E$ edges are place at random in the $N(N-1) / 2$ possible positions, then the probability that there is an edge between two nodes is

$$
\operatorname{Prob}(E \text { Edges for } N \text { Nodes })=\frac{E}{N(N-1) / 2}
$$

For MUMS networks, the time dependant edge metric is:

$$
E(t)=\text { number of edges at time } t
$$

To assess if a MUMS network (at any given time) has a significant difference in the number of edges, one can then compute the number of standard deviations by which the network under consideration deviates from random at time $t$.

\subsection{Specific Metrics}

The metrics presented in Section 3.1 can be expanded and quantified so as to provide more specific metrics associated with MUMS networks. An initial collection of such metrics is presented in Table 2. Also provided in Table 2 is a list of questions that the metrics are intended to assess. By developing such metrics, an initial step results in attempting to quantify future manned and unmanned systems interactions.

\section{CONCLUSIONS}

This paper presented preliminary metrics to support understanding the extent of interactions that would arise in a system of cooperating manned and unmanned systems (MUMS). The metrics seek to help explain if attachments are localized to specific situations or if they are more pervasive throughout a MUMS society. Such metrics can be applied to multiple, collaborating, and interacting manned and robotic systems when they are represented as a collection of dynamic, interacting nodes. As robots become more and more intelligent, they will be able to support increased collaborative interactions with each other and with people, where rather than being commanded by people with continual oversight, they will truly interact. This paper provides an initial approach and quantifying such interactions.

\section{REFERENCES}

[Aie] W. Aiello, F. Chung, and L. Lu, "A Random Graph Model for Massive Graphs," in [New], p259-268.

[Alo] U. Alon, An Introduction to Systems Biology, Design Principles of Biological Circuits, Chapman \& Hall/CRC, 2007.

[Bro] A. Broder, et al., "Graph Structure in the Web," Computer Networks, 33 (2000) 309-320.

[Bre] C. Breazeal, "Social Interactions in HRI: The Robot View," IEEE Trans. on Man, Cybernetics, and Sys - Part C, 2003.

[deS] D.de Solla Price, "Networks of Scientific Papers," Science vol. 149, in [New], p. 129-154.

[Erd] P. Erdos and A. Renyi, "On the Evolution of Random Graphs," A Mathematickai Kutato Intezet Kozlemenyei, in [New], p38-61.

[GT] Georgia Tech Research Horizons Magazine, June 8, 2007, From Science Fiction to Reality: Personal Robots Emerge to Improve Quality of Life at Work, Home, and School.

[Hin] P. Hinds, T. Roberts, H. Jones, "Whose Job Is It Anyway? A Study of Human-Robot Interaction in a Collaborative Task," Human-Computer Interaction, 2004, Vol 19, pp. 151181, 2004.

[Kie] S. Kiesler and P. Hinds, "Introduction to This Special Issue of Human-Robot Interaction", Human-Computer Interaction, 2004, Vol 19, pp. 1-8, 2004.

[New] M. Newman, A-L Barabasi, D.J. Watts (eds), The Structure and Dynamics of Networks, Princeton Univ. Press, 2006.

[New2] M. Newman, "The Structure and Function of Complex Networks," SIAM Review, Vol. 54, No 2, p167-256, 2003.

[Tra] J. Travers, and S. Milgram, “ An Experimental Study of the Small World Problem,” in [New], p 130-148.

[Wat] D. J. Watts, S. H. Strogatz, "Collective Dynamics of 'Small-World' Networks,” Nature, vol. 393, 4 Jun 1998.

[Wei] L.G. Weiss, “A Framework for Co-operating Manned and Unmanned Systems, Part I - Framework Description,” (draft)

Permission to make digital or hard copies of all or part of this work for personal or classroom use is granted without fee provided that copies are not made or distributed for profit or commercial advantage, and that copies bear this notice and the full citation on the first page. To copy otherwise, to republish, to post on servers or to redistribute to lists, requires prior specific permission and/or a fee. PerMIS'10, September 28-30, 2010, Baltimore, MD, USA. Copyright (c) 2010 
Table 2. Specific MUMS Metrics

\begin{tabular}{|c|c|c|}
\hline Metric / Method & Description & Question it Answers \\
\hline $\begin{array}{l}\text { Collaborative } \\
\text { Filtering }\end{array}$ & $\begin{array}{l}\text { - Technique To Predict New Likes/Dislikes Based On An Individual's Other } \\
\text { Preferences (Captures Preferential Attachment) } \\
\text { - Used In Targeted Advertising (person who bought } x \text { also bought } y \text { ) } \\
\text { - Build an item-item matrix determining relationships between nodes } \\
\text { - Using the matrix, and data on the current node, predict its future state }\end{array}$ & $\begin{array}{l}\text { Is Preferential Attachment } \\
\text { Better than Diverse } \\
\text { Attachment? }\end{array}$ \\
\hline $\begin{array}{l}\text { Clustering } \\
\text { Coefficient }\end{array}$ & $\begin{array}{l}\text { - Probability That Two Nodes That Are Connected To The Same Node Will } \\
\text { Be Connected (Probability That A Friend Of A Friend Is A Friend) } \\
\text { - At Node } i, \quad \mathrm{C} i=\frac{\# \text { Connected Nbr Pairs, }}{1 / 2 k(k-1)} \text { where } k=\text { degree of node } i \\
\text { - } \mathrm{C}=\text { Average of } \mathrm{C} i \\
\text { - } \text { Social Networks, } \mathrm{C}=\mathrm{O}(1), \text { Large } \mathrm{n} \\
\text { - Random Graphs, } \mathrm{C}=\mathrm{O}(1 / \mathrm{n}), \text { Large } \mathrm{n}\end{array}$ & $\begin{array}{l}\text { Does Clustering Occur in } \\
\text { Dynamic MUMS Networks? }\end{array}$ \\
\hline Assortive Mixing & $\begin{array}{l}\text { - Which Nodes Pair Up With Others (e.g., mating choices, geography) } \\
\text { - } \quad E \text { et } E i j=\# \text { edges that connect nodes of type } i \text { to nodes of type } j \\
\text { - } E=[E i j], e=E /\|E\|=\text { normalized mixing matrix } \\
\text { - } \quad P(j=\text { fraction of edges that fall between nodes of type } i \text { and } j \\
\text { Node of Type } i \\
\text { - Let } r=\frac{T r e-\|e 2\|}{1-\|e 2\|} \\
\text { - If } \mathrm{r}=1 \text {, every edge connects to same type of node } \\
\text { - If } r=0, \text { randomly mixed network } \\
\text { - Otherwise, some level of mixing exists }\end{array}$ & $\begin{array}{l}\text { Can We Calculate } r \text { for a } \\
\text { MUMS Network? }\end{array}$ \\
\hline $\begin{array}{l}\text { Degree } \\
\text { Correlations }\end{array}$ & $\begin{array}{l}\text { - Mixing According to the Degree, } k \text {, of the Node } \\
\text { - } \quad \text { Can Give Rise to Interesting Network Structures } \\
\text { - If Correlation Increases with } k=>\text { Assortive Mixing } \\
\text { - If Correlation Decreases with } k=>\text { Disassortive Mixing } \\
\text { - Social Networks are Assortive } \\
\end{array}$ & $\begin{array}{l}\text { Are MUMS Environments } \\
\text { Assortive or Disassortive? }\end{array}$ \\
\hline $\begin{array}{l}\text { Degree } \\
\text { Distributions }\end{array}$ & $\begin{array}{ll}\text { - } & \mathrm{P}(k)=\text { Probability That A Node Chosen At Random Has Degree } k \\
\text { - } & \text { Random Graphs Have Poisson/Binomial Distributions, large } n \\
\text { - } & \text { Social Networks Are Right Skewed } \\
\text { - } & \text { When } \mathrm{P}(k)=k-\alpha, \text { System Follows a Power Law } \\
\text { - } & \alpha \text { Constant }=>\text { Scale-free Network Results } \\
\end{array}$ & $\begin{array}{l}\text { Do MUMS Environments } \\
\text { Attain a Scale-Free } \\
\text { Network? }\end{array}$ \\
\hline $\begin{array}{l}\text { Network } \\
\text { Resilience, Large n }\end{array}$ & $\begin{array}{ll}\text { - } & \text { Node Removal - May Loose Robustness but Not Functionality } \\
\text { - } & \text { Random Node Removal => Little Effect } \\
\text { - } & \text { Targeted Node Removal => Large Effect }\end{array}$ & $\begin{array}{l}\text { How Do Node Failures } \\
\text { Affect MUMS Networks? }\end{array}$ \\
\hline $\begin{array}{l}\text { Network } \\
\text { Navigation }\end{array}$ & $\begin{array}{l}\text { - The } 6 \text { Degree of Separation Model Showed Short Paths Exists } \\
\text { - It Also Showed that People are Good at Finding Them Without Knowledge } \\
\text { of the Network or Connectivity } \\
\text { - Not True for Random Graphs } \\
\text { - Without A Means To Estimate Social Distance, Individuals Are Unable To } \\
\text { Move A Message Closer To A Remote Target }\end{array}$ & $\begin{array}{l}\text { Can We Merge Artificial and } \\
\text { Social Networks to Enable } \\
\text { Efficient Navigation within } \\
\text { MUMS Networks? }\end{array}$ \\
\hline $\begin{array}{l}\text { Models of } \\
\text { Network Growth }\end{array}$ & 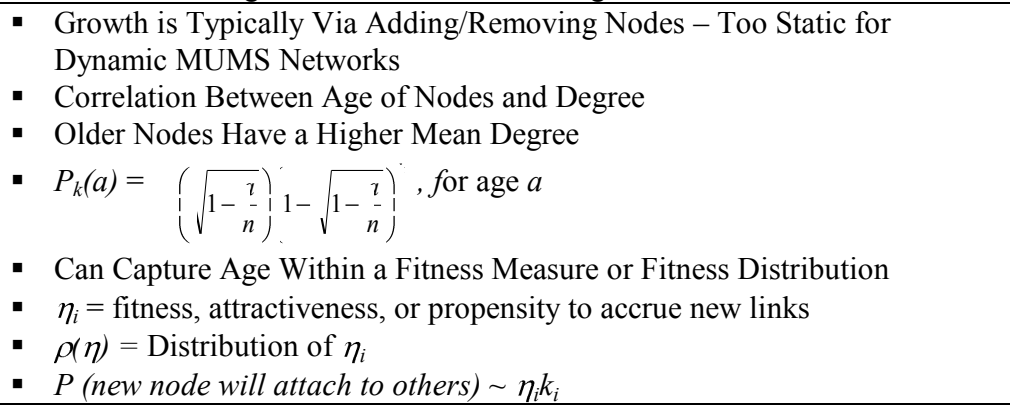 & $\begin{array}{l}\text { What Patterns or Statistical } \\
\text { Regularities May Result in } \\
\text { MUMS Networks? }\end{array}$ \\
\hline
\end{tabular}

Permission to make digital or hard copies of all or part of this work for personal or classroom use is granted without fee provided that copies are not made or distributed for profit or commercial advantage, and that copies bear this notice and the full citation on the first page. To copy otherwise, to republish, to post on servers or to redistribute to lists, requires prior specific permission and/or a fee. PerMIS'10, September 28-30, 2010, Baltimore, MD, USA. Copyright (c) 2010 